\title{
Effects of n-3 polyunsaturated fatty acids on thrombogenic risk markers in subjects with moderate risk for CVD
}

\author{
E. Bozbas ${ }^{1}$, R. Zhou ${ }^{1}$, K. Allen-Redpath ${ }^{1}$ and P. Yaqoob ${ }^{1}$ \\ ${ }^{1}$ Hugh Sinclair Unit of Human Nutrition, University of Reading, Whiteknights, Reading, UK
}

This abstract was presented as the Whole Body Metabolism Theme highlight.

Fish oil is suggested to have a role in the prevention of cardiovascular disease (CVD) with much of the research on modulation of risk factors that contribute to the development of $\mathrm{CVDs}^{(1)}$. Although the exact underlying mechanism by which fish oil improves cardiac health is not clear, these cardioprotective effects could be associated with the high n-3 polyunsaturated fatty acid (PUFA) content in fish oil, particularly eicosapentaenoic acid (EPA) and docosahexaenoic acid (DHA), and their beneficial effects on cardiovascular risk markers ${ }^{(2)}$. Concerning thrombogenic risk markers, some trials point to beneficial effects of $n-3$ PUFAs on vascular health and function $^{(3)}$, endothelial function ${ }^{(4)}$, and thrombosis by favourable effects on platelet aggregation, hypercoagulation, fibrinolysis, and thrombin generation. However, there is a lack of clarity regarding the effects of n-3 PUFA on thrombogenic risk markers and this requires investigation in a well-designed study. Therefore, the objective was to investigate whether the effect of daily supplementation with fish oil-derived n-3 PUFA altered thrombogenic markers, including platelet aggregation, coagulation, fibrinolysis, and thrombin generation, in subjects with moderate risk for CVD.

The HI-FIVE Study was a randomised, double-blind, placebo-controlled crossover intervention study. Forty subjects aged 40-70y with moderate risk of CVDs were supplemented with capsules containing either fish oil (1.8 g/d n-3 PUFA) or placebo (high-oleic safflower oil) for a 12-week period. Platelet aggregation was assessed by 96-well plate-based aggregometry. Fibrin properties were investigated by assessment of coagulation and fibrinolysis using a thrombodynamics analyser (Hemacore) by addition of tissue-type plasminogen activator (tPA, $4 \mathrm{nM}$ ) to platelet-free plasma. Thrombin formation was measured using tissue factor-induced, platebased thrombin generation assay (Technothrombin TGA kit), which assesses a change in fluorescence as a result of cleavage of a fluorogenic substrate by thrombin over time upon activation of the clotting cascade by tissue factor.

The intervention had no effect on platelet aggregation in response to adenosine diphosphate (ADP), cross-linked collagen-related peptide (CRP-XL), epinephrine, thrombin receptor-activating peptide-6 (TRAP-6) or U46619. Fish oil supplementation significantly decreased the rate of clot growth and clot size at $30 \mathrm{~min}(\mathrm{p}<0.05)$, but there was no effect on fibrinolysis parameters, including lysis onset time or lysis progression. Fish oil significantly decreased peak thrombin generation, time to reach peak thrombin generation, velocity index and area under the curve for thrombin generation over $1 \mathrm{~h}$, and also resulted in prolonged lag time for thrombin generation $(\mathrm{p}<0.001)$.

This well-designed study supports and advances existing evidence regarding the beneficial effects of n-3 PUFAs on thrombogenic risk factors. N-3 PUFA significantly reduced the formation of a fibrin clot and thrombin generation, which reflect a beneficial effect on procoagulant potential, but did not influence clot breakdown or platelet function.

\section{References}

1. Baker EJ, Yusof MH, Yaqoob P, et al. (2018) Mol Aspects Med, 169-181.

2. Calder PC (2014) Eur J Lipid Sci Technol, 1280-1300.

3. Wu JH, Lemaitre RN, King IB, et al. (2012) Circulation, 1084-1093.

4. Renga B \& Scavizzi F (2017) Acta Cardiol, 2-8. 